ACCEPTED MANUSCRIPT • OPEN ACCESS

\title{
Multiply-excited states and their contribution to opacity in CO2 laser- driven tin-plasma conditions
}

To cite this article before publication: John Sheil et al $2020 \mathrm{~J}$. Phys. B: At. Mol. Opt. Phys. in press https://doi.org/10.1088/1361-6455/abcedf

\section{Manuscript version: Accepted Manuscript}

Accepted Manuscript is "the version of the article accepted for publication including all changes made as a result of the peer review process, and which may also include the addition to the article by IOP Publishing of a header, an article ID, a cover sheet and/or an 'Accepted Manuscript' watermark, but excluding any other editing, typesetting or other changes made by IOP Publishing and/or its licensors"

This Accepted Manuscript is @ $\mathbf{2 0 2 0}$ The Author(s). Published by IOP Publishing Ltd.

As the Version of Record of this article is going to be / has been published on a gold open access basis under a CC BY 3.0 licence, this Accepted Manuscript is available for reuse under a CC BY 3.0 licence immediately.

Everyone is permitted to use all or part of the original content in this article, provided that they adhere to all the terms of the licence https://creativecommons.org/licences/by/3.0

Although reasonable endeavours have been taken to obtain all necessary permissions from third parties to include their copyrighted content within this article, their full citation and copyright line may not be present in this Accepted Manuscript version. Before using any content from this article, please refer to the Version of Record on IOPscience once published for full citation and copyright details, as permissions may be required. All third party content is fully copyright protected and is not published on a gold open access basis under a CC BY licence, unless that is specifically stated in the figure caption in the Version of Record.

View the article online for updates and enhancements. 


\title{
Multiply-excited states and their contribution to opacity in $\mathrm{CO}_{2}$ laser-driven tin-plasma conditions
}

\author{
J. Sheil ${ }^{1 *}$ and O. O. Versolato ${ }^{1,2}$ \\ ${ }^{1}$ Advanced Research Center for Nanolithography, Science Park 106, 1098 XG Amsterdam, The Netherlands and \\ ${ }^{2}$ Department of Physics and Astronomy, and LaserLaB, Vrije Universiteit, \\ De Boelelaan 1081, 1081 HV Amsterdam, The Netherlands \\ A. J. Neukirch and J. Colgan ${ }^{\dagger}$ \\ Los Alamos National Laboratory, Los Alamos, NM 87545, USA
}

\begin{abstract}
A recent study [Nat. Commun. 11, 2334 (2020)] has found that transitions between multiplyexcited configurations in open $4 d$-subshell tin ions are the dominant contributors to intense EUV emission from dense, Nd:YAG-driven (laser wavelength $\lambda=1.064 \mu \mathrm{m}$ ) tin plasmas. In the present study, we employ the Los Alamos Aтоміс code to investigate the spectral contribution from these transitions under industrially-relevant, $\mathrm{CO}_{2}$ laser-driven $(\lambda=10.6 \mu \mathrm{m})$ tin plasma conditions. First, we employ Busquet's ionisation temperature method to match the average charge state $\langle Z\rangle$ of a nonlocal-thermodynamic equilibrium (non-LTE) plasma with an LTE one. This is done by varying the temperature of the LTE calculations until a so-called ionisation temperature $T_{Z}$ is established. Importantly, this approach generates LTE-computed configuration populations in excellent agreement with the non-LTE populations. A corollary of this observation is that the non-LTE populations are well-described by Boltzmann-type exponential distributions having effective temperatures $T_{\text {eff }} \approx T_{Z}$. In the second part of this work, we perform extensive level-resolved LTE opacity calculations at $T_{Z}$. It is found that $66 \%$ of the opacity in the industrially-relevant $2 \%$ bandwidth centered at $13.5 \mathrm{~nm}$ arises from transitions between multiply-excited states. These results reinforce the need for the consideration of complex, multiply-excited states in modelling the radiative properties of laser-driven plasma sources of EUV light.
\end{abstract}

\section{INTRODUCTION}

Powerful sources of short-wavelength radiation play a crucial role in many high-profile scientific and industrial endeavours [1]. Examples include high-resolution biological imaging [2-4], materials science $[5,6]$ and the manufacturing of integrated circuits (ICs) in the semiconductor industry $[7,8]$. The use of short-wavelength radiation in this latter application is expected to enable the printing of sub-10 nm feature sizes on ICs [9], thus sustaining the production of cheaper and more powerful computer chips into the future.

The key technology driving feature-size miniaturisation in the semiconductor industry today is extreme ultraviolet lithography (EUVL) $[10-13]$. As the name suggests, this technology uses EUV light to print features on ICs. In an industrial setting, this EUV light is generated in a laser-produced plasma (LPP) formed by irradiating tin microdroplets with $\mathrm{CO}_{2}$ laser light $(\lambda=10.6$ $\mu \mathrm{m})[14,15]$. The ensuing laser-droplet interaction generates a plasma having moderate electron temperatures and densities $\left(T_{e} \approx 20-40 \mathrm{eV}, n_{e} \approx 10^{18}-10^{19} \mathrm{~cm}^{-3}\right)$ comprising large populations of $\mathrm{Sn}^{8+}-\mathrm{Sn}^{14+}$ ions. Spectra recorded from such plasmas are known to exhibit an intense, narrowband feature peaked near $13.5 \mathrm{~nm}$ [1620]. Importantly, this emission feature provides optimum

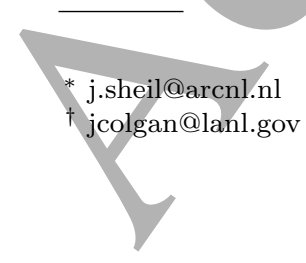

wavelength overlap with the $2 \%$ reflective bandwidth centered at $13.5 \mathrm{~nm}$ defined by molybdenum/silicon multilayer mirrors (MLMs) employed in lithography tools [12]. This combination of (i) optimum emission characteristics and (ii) a debris-limited droplet-target (key for minimising MLM contamination from plasma expansion [21]) established tin LPPs as the light source of choice for EUVL. In fact, after more than 20 years of research and development, EUVL has now entered the realm of high-volume manufacturing of new-generation ICs [22].

The aforementioned intense, narrowband emission feature observed in laser-produced tin plasmas has, to date, been predominantly attributed to overlapping $\Delta n=0$ transition arrays of the form $4 p^{6} 4 d^{m}-\left(4 p^{5} 4 d^{m+1}+\right.$ $\left.4 p^{6} 4 d^{m-1} 4 f\right)$ in charge states $\mathrm{Sn}^{8+}-\operatorname{Sn}^{14+}(m=$ $6-0)$ [23-25]. Such claims have been supported by charge-state-resolved spectroscopic experiments [26-29] as well as numerous theoretical efforts employing advanced atomic structure codes [30-39]. Although serving as the general consensus for many years, a recent study of Torretti et al. has challenged this viewpoint [13]. In the most detailed tin-ion opacity calculations performed to-date, it was found that transitions between multiply-excited configurations in $\mathrm{Sn}^{11+}-\mathrm{Sn}^{14+}$ are in fact the dominant contributors to EUV emission in Nd:YAG-driven $(\lambda=1.064 \mu \mathrm{m})$ tin LPPs. Surprisingly, the well-known $4 p^{6} 4 d^{m}-\left(4 p^{5} 4 d^{m+1}+4 p^{6} 4 d^{m-1} 4 f\right)$ transitions play only a minor role; they comprise $11 \%$ of the opacity in the $5-20 \mathrm{~nm}$ region and $19 \%$ of the opacity in the $2 \%$ bandwidth. Remarkably, it is transitions between complex, multiply-excited configurations (doubly-, 
triply- and quadruply-excited configurations) which dominate the opacity spectrum. Strong support for their spectroscopic dominance was evidenced through comparisons of experimental tin-droplet EUV spectra with onedimensional radiation transport simulations. The incorporation of multiply-excited transitions in these simulations was deemed crucial for the reproduction of the experimental spectra.

The purpose of the present paper is to elucidate the role of multiply-excited states in $\mathrm{CO}_{2}$-driven tin plasmas which are currently used in the industry to provide light for EUVL. The main difference between the present work and previous atomic modelling work of Sasaki and co-authors [37, 40, 41] is the extensivity of the atomic structures employed. For example, in Ref. $[37,40,41]$, a less extensive number of doublyexcited states were included in their atomic models. Their inclusion was mainly for the purpose of understanding satellite transition array emission of the form $4 p^{6} 4 d^{m-1} n l-\left(4 p^{5} 4 d^{m} n l+4 p^{6} 4 d^{m-2} 4 f n l\right)$. The configuration sets adopted in the present work include large numbers of doubly-, triply- and quadruply-excited configurations and are therefore significantly more extensive than those considered previously. Importantly, we find that transitions between multiply-excited states make a substantial contribution to the opacity in the $5-20 \mathrm{~nm}$ region in plasma conditions pertinent to those generated in $\mathrm{CO}_{2}$ laser-driven tin plasmas.

The current paper is organised as follows: In section II, we describe the atomic structure and opacity calculations performed with the Los Alamos suite of atomic physics and population kinetics codes. An examination of non-LTE- and LTE-computed tin ionisation distributions and configuration populations in $\mathrm{CO}_{2}$ laser-driven plasma conditions follows in section III. In section IV we present level-resolved LTE-computed opacities of $\mathrm{Sn}^{11+}$ $-\mathrm{Sn}^{14+}$. Finally, a summary of this work is given in section $\mathrm{V}$.

\section{ATOMIC STRUCTURE AND OPACITY CALCULATIONS}

In the present work, we have employed the Los Alamos National Laboratory (LANL) suite of atomic structure and collision codes [42] as well as the population kinetics code Aтоміс [43, 44] to calculate the opacities of a tin plasma at densities and temperatures relevant to those attained in a $\mathrm{CO}_{2}$ laser-driven plasma. Given the central role that these codes play in this study, we devote the following subsections to their description.

\section{A. Atomic structure calculations}

The first step in the present opacity calculations is the generation of all relevant atomic data (energy levels, oscillator strengths, ionisation potentials, etc.). In Torretti et al. [13], considerable effort was devoted to benchmarking the calculated atomic structures with available experimental data and therefore we have utilised these atomic structures in the present study. In brief, the CATS code [45] was employed in the calculation of level energies and absorption oscillator strengths. This code is based on Cowan's original atomic structure code [46, 47] and exhibits a favourable feature where one can scale the magnitude of the radial integrals in order to bring theoretical level structures into excellent agreement with experimental observations. This semi-empirical approach has proven to be an indispensable tool in atomic spectroscopy, especially for systems exhibiting complicated level structures $[29,48-51]$. In the work of Torretti et al. [13], optimum agreement between atomic structure calculations and previous experimental observations $[17,26,27]$, was found by applying scaling factors of $0.87 / 0.75$ to the radial integrals associated with singly/multiply-excited energy levels. These two scaling factors were adopted for all charge states considered in this work. Extensive configuration sets were employed in the calculations through use of the so-called "2-mode" approach. In these calculations, configuration interaction (CI) was retained for a/large number of configurations associated with $\Delta n=0$ and $\Delta n=1$ single, double and triple excitations from the $n=4$ manifold of the ground-state configuration. Additional configurations were included using intermediate coupling (only interactions between levels of the same configuration are considered) [39]. This approach retains a significant level of detail provided by full CI calculations however at a much reduced computational cost.

\section{B. Opacity calculations}

Knowledge of opacities is crucial for understanding radiation transport in a host of plasma environments, such as those encountered in stellar interiors, fusion devices and short-wavelength plasma light sources [30, 52-55]. In this context, an opacity quantifies how photons, travelling in a plasma, are absorbed and scattered through interactions with the plasma constituents. For a given photon frequency $\nu$, the opacity $\kappa_{\nu}^{\mathrm{T}}$ is defined as the contribution of four mechanisms:

$$
\kappa_{\nu}^{\mathrm{T}}=\kappa_{\nu}^{\mathrm{BB}}+\kappa_{\nu}^{\mathrm{BF}}+\kappa_{\nu}^{\mathrm{FF}}+\kappa_{\nu}^{\mathrm{SCAT}} .
$$

The first three terms represent bound-bound (photoabsorption), bound-free (photoionisation) and free-free (inverse bremsstrahlung) contributions to the opacity and each contain a factor correcting for stimulated emission. The final term represents photon scattering. In the present work, we are mainly concerned with the boundbound contribution to the opacity (since all other contributions are minor in the wavelength range of interest for these plasma conditions) and therefore we drop the 
superscript "BB" notation in the following. The boundbound opacity $\kappa_{\nu}$ is written

$$
\kappa_{\nu}=\rho^{-1} \sum_{i<j} \sigma_{\nu}^{i j}\left(n_{i}-g_{i} g_{j}^{-1} n_{j}\right),
$$

where $\rho$ is the mass density and the summation term is known as the absorptivity. Here, $\sigma_{\nu}^{i j}$ is the cross section for photoabsorption from level $i$ to level $j$ and $n_{i(j)}$ are the population densities (simply called "populations" in the following) of energy levels $i(j)$ having statistical weights $g_{i(j)}$. The sum runs over all transitions $i \rightarrow j$ associated with photon absorption at frequency $\nu$. The second term in the summation is the aforementioned correction for stimulated emission. The cross section for photoabsorption $\sigma_{\nu}^{i j}$ is given by

$$
\sigma_{\nu}^{i j}=\frac{\pi e^{2}}{m_{e} c} f_{i j} \phi(\nu)
$$

where $e, m_{e}$ and $c$ are the electron charge, electron mass and speed of light, respectively. The term $f_{i j}$ is the absorption oscillator strength for a transition $i \rightarrow j$ and $\phi(\nu)$ is the line profile function satisfying $\int_{0}^{\infty} \phi(\nu) d \nu=1$.

As one can see from Eqs. (2) and (3), evaluation of the bound-bound opacity requires knowledge of both atomic structure $\left(\nu\right.$ and $\left.f_{i j}\right)$ and the level populations $n_{i(j)}$. Evaluation of this latter quantity requires knowledge of the plasma environment in which the atomic species is embedded. Aтоміс, using as input the mass density $\rho$ and electron temperature $T_{e}$, can calculate these level populations in LTE or non-LTE plasma conditions (the radiation temperature $T_{r}$ can also be specified in non-LTE calculations). The LTE-mode of ATOMIC uses the ChemEOS equation-of-state (EOS) model to derive level populations and charge state distributions (CSDs) $[56,57]$. This model is based on the free-energyminimisation approach and the occupation probability formalism (this latter procedure allows plasma density effects to be included in a consistent manner). Importantly, non-ideal plasma effects, such as plasma microfields and strong coupling are included in the free-energy term. This leads to modified Boltzmann and Saha relations from which level populations and CSDs are derived.

ATOMIC also has the capability of modelling plasmas under non-LTE conditions $[58,59]$. Unlike LTE plasmas, the calculation of level populations in non-LTE conditions requires a microscopic viewpoint in which the rates of all relevant atomic populating and depopulating processes are calculated. The most general definition of a non-LTE plasma is one which exhibits any characteristic departure from LTE conditions, such as the existence of a non-Maxwellian electron energy distribution function (EEDF) or a non-Planckian radiation field. As such, there are a variety of ways that a plasma can be defined as under non-LTE conditions. Consider an atomic state (energy level, configuration or superconfiguration) $i$ associated with some charge state $\zeta$ in the plasma. In non-LTE plasmas, the population of this state evolves according to a collisional-radiative (CR) equation of the form $[60,61]$

$$
\frac{d n_{i \zeta}}{d t}=\sum_{j \zeta^{\prime}} n_{j \zeta^{\prime}} R_{j \zeta^{\prime} \rightarrow i \zeta}-n_{i \zeta} \sum_{j \zeta^{\prime}} R_{i \zeta \rightarrow j \zeta^{\prime}}
$$

Here, $R_{j \zeta^{\prime} \rightarrow i \zeta}=R_{j \zeta^{\prime} \rightarrow i \zeta}^{\mathrm{C}}+R_{j \zeta^{\prime} \rightarrow i \zeta}^{\mathrm{R}}$ denotes the rate of collisional $\left(R_{j \zeta^{\prime} \rightarrow i \zeta}^{\mathrm{C}}\right)$ and radiative $\left(R_{j \zeta^{\prime} \rightarrow i \zeta}^{\mathrm{R}}\right)$ processes connecting $j$ (associated with some charge state $\zeta^{\prime}$ ) to $i$. In a similar fashion, rates describing $i \rightarrow j$ are denoted $R_{i \zeta \rightarrow j \zeta^{\prime}}$. The first term in Eq. (4) represents population influx into $i$ and the second term describes population outflux from $i$. A CR equation of the form Eq. (4) exists for all atomic states $i$ in each charge state $\zeta$ present in the plasma. Coupling, these states yields the so-called rate equation

$$
\sqrt{ } \frac{d \mathbf{N}}{d t}=A \mathbf{N} .
$$

Here, $\mathbf{N}$ is a vector of atomic state populations $\left(n_{i \zeta} \in\right.$ $\mathbf{N})$ and $A$ is the so-called rate matrix containing all relevant rates $R_{j \zeta^{\prime} \rightarrow i \zeta, i \zeta \rightarrow j \zeta^{\prime}}^{\mathrm{C}, \mathrm{R}}$.

The choice of atomic processes to be included in the rate matrix $A$ is highly case-dependent; in coronal plasmas, for example, one can neglect the role of threebody recombination. Such simplifications are not possible in the current case and therefore we must consider a wide range of atomic processes (see section III for more details). Fortunately, AтоміC has the capability of modelling a variety of (de-)populating processes such as electron-impact excitation/ionisation, photoexcitation, photoionisation, autoionisation (AI), etc. Cross sections for electron-impact excitation were calculated in the plane-wave Born (PWB) approximation using the CATS code. Electron-impact ionisation cross-sections were obtained from the GIPPER code (photoionisation cross sections can also be obtained if an external radiation field is included in the non-LTE model) [62]. Атоміс uses these cross sections to calculate the rates which form the entries of $A$. Invoking a conservation equation of the form

$$
\sum_{\zeta=0}^{Z} \sum_{i=0}^{N_{i}-1} n_{i \zeta}=n_{i o n}
$$

where $N_{i}$ is the number of atomic states and $n_{\text {ion }}$ is the ion density allows one to solve Eq. (5) for the atomic state populations $n_{i \zeta}$. These populations can then used to produce opacities (Eq. (2)) in a similar fashion as in the LTE mode of ATOMic.

Finally, it should be emphasized that a consistent atomic structure has been employed in all stages of the 
calculation, ranging from the evaluation of collisional cross-sections to the derivation of atomic state populations and level-resolved opacities. This procedure ensures self-consistency in all aspects of the calculation.

\section{TIN-ION OPACITIES IN $\mathrm{CO}_{2}$ LASER-DRIVEN PLASMA CONDITIONS}

The study of $\mathrm{CO}_{2}$ laser irradiation of tin targets comprises a large body of work (see [20] and references therein). A very important aspect of this work is the study of tin-ion opacities in the relevant plasma conditions [32, 37-39, 63]. Plasmas generated by $\mathrm{CO}_{2}$ laser irradiation lie in a rather unique position in $\left(n_{e}, T_{e}\right)$ space; electron densities are not high enough to allow for LTE conditions to prevail, however not so low to justify the use of coronal equilibrium relations. In this respect, the atomic modelling of $\mathrm{CO}_{2}$-driven laser-plasmas should, in theory, employ the CR approach described in section II.

As outlined in the Introduction, the goal of the present work is to evaluate the role of multiply-excited states in $\mathrm{CO}_{2}$ laser-driven plasma conditions. As such, we must inherit the complicated level structures considered in Ref. [13]. As discussed in Ref. [13, 39], the use of extensive atomic models for $\mathrm{Sn}^{11+}-\mathrm{Sn}^{14+}$ is necessary to ensure (i) correct wavelength positions of $\Delta n=0$ transitions (which are sensitive to the choice of underlying atomic structure as a result of CI) and (ii) partition function convergence [56]. Unfortunately, this choice of extensivity over simplicity makes the solution of Eq. (5) for these level structures intractable; there are simply too many atomic levels to consider. As discussed in Ref. [13], the calculation of detailed, level-resolved opacities for such complex structures is currently only possible using the LTE approach in Aтомic.

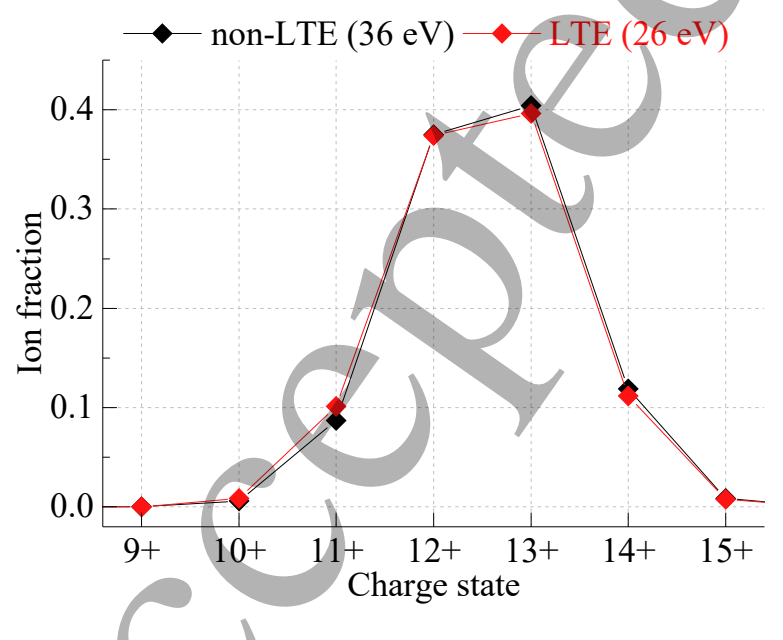

FIG. 1. Charge-state distributions for plasmas calculated in non-LTE $\left(T_{e}=36 \mathrm{eV}\right.$; shown in black) and LTE $\left(T_{Z}=26\right.$ $\mathrm{eV}$; shown in red) conditions. The plasma mass density used in both calculations was $\rho=2 \times 10^{-4} \mathrm{~g} \mathrm{~cm}^{-3}$.

\section{A. Busquet's ionisation temperature method}

In order to circumvent computational intractabilities associated with level-resolved, non-LTE opacity calculations involving multiply-excited configurations, we have employed the ionisation temperature method of Busquet $[64,65]$ to effectively "mimick" non-LTE opacities using LTE computations. The key observation underlying this method is that non-LTE CSDs (performed at some $T_{e}$ ) often resemble LTE CSDs undertaken at a temperature $T_{Z}$ different to $T_{e}[66]$. The first step in Busquet's approach is to identify the LTE temperature $T_{Z}$ which yields optimum agreement in average charge state $\langle Z\rangle$ between the non-LTE and LTE calculations, i.e.,

$$
\left\langle Z\left(T_{e}\right)\right\rangle_{\mathrm{non}-\mathrm{LTE}}=\left\langle Z\left(T_{Z}\right)\right\rangle_{\mathrm{LTE}} \text {. }
$$

In the current context, $T_{Z}$ is known as the "ionisation temperature". Once $T_{Z}$ is determined, one then performs detailed, level-resolved LTE opacity calculations at $T_{Z}$. Higher-order moments of the CSD, such as the width, are not considered in this method. Although this approach provides a numerically cost-efficient means of incorporating non-LTE opacities (and equation-of-state quantities) into hydrodynamic codes, Klapisch and Bar-Shalom have stressed that it should only be applied to plasmas not far from pure LTE conditions [66].

We wish to test the validity this method in the context of the current problem. Guided by previous modelling efforts of Sasaki and co-workers [37, 40, 41], we have undertaken a non-LTE configuration-averaged (CA) AтоміC calculation at $\rho=2 \times 10^{-4} \mathrm{~g} \mathrm{~cm}^{-3}$ and $T_{e}=36 \mathrm{eV}$. The electron density of this plasma is $n_{e}=1.27 \times 10^{19} \mathrm{~cm}^{-3}$, close to the critical electron density for $\mathrm{CO}_{2}$ laser light $\left(n_{\text {crit }, \mathrm{CO}_{2}}=1.1 \times 10^{19} \mathrm{~cm}^{-3}\right)$. The $\mathrm{CA}$ approach generates appropriately averaged energies, oscillator strengths and PWB electron-impact excitation cross sections from which configuration-to-configuration rates are derived [59]. This approach is employed in the present context to ensure computational tractability. Configurations sets employed in the CA CATS calculations for $\mathrm{Sn}^{12+}$ and $\mathrm{Sn}^{14+}$ are provided in Table I. The calculations were performed under steady-state conditions, i.e., $d \mathbf{N} / d t=0$. This approximation is valid for plasmas where the time taken to generate a given CSD (through ionisation and recombination processes) occurs on timescales shorter than the laser pulse duration. This is certainly true for industrial LPP EUV sources, which are typically driven by multi-10-nanosecond-long $\mathrm{CO}_{2}$ pulses. We calculated the rates of electron-impact excitation/ionisation and autoionisation (as well as their inverse processes) assuming a Maxwell-Boltzmann EEDF at $T_{e}=36 \mathrm{eV}$. The calculations were performed without an external radiation field, i.e., photoexcitation and photoionisation processes were not considered (see subsection B below for further discussion). The process of spontaneous emission was included in the model. 
1

2

3

4

5

6

7

8

9

\begin{tabular}{ll}
\hline \hline $\mathrm{Sn}^{12+}$ & $\mathrm{Sn}^{14+}$ \\
\hline Ground configuration & Ground configuration \\
$4 s^{2} 4 p^{6} 4 d^{2}$ & $4 s^{2} 4 p^{6}$ \\
Singly-excited configurations & Singly-excited configurations \\
$4 s^{2} 4 p^{6} 4 d+\{4 f, 5 l\}$ & $4 s^{2} 4 p^{5}+\{4 d, 4 f, 5 l\}$ \\
$4 s^{2} 4 p^{5}+\left\{4 d^{3}, 4 d^{2} 4 f, 4 d^{2} 5 l\right\}$ & $4 s^{1} 4 p^{6}+\{4 d, 4 f, 5 l\}$ \\
$4 s 4 p^{6}+\left\{4 d^{3}, 4 d^{2} 4 f, 4 d^{2} 5 l\right\}$ & \\
Doubly-excited configurations & Doubly-excited configurations \\
$4 s^{2} 4 p^{6}+\left\{4 f^{2}, 4 f 5 l, 5 s 5 l, 5 p 5 l^{\prime}, 5 d 5 l, 5 f 5 g\right\}$ & $4 s^{2} 4 p^{4}+\left\{4 d^{2}, 4 d 4 f, 4 f^{2}, 4 d 5 l, 4 f 5 l, 5 s 5 l, 5 p 5 l^{\prime}, 5 d 5 f, 5 d 5 g, 5 f 5 g\right\}$ \\
$4 s^{2} 4 p^{5}+\left\{4 d 4 f^{2}, 4 d 5 s^{2}, 4 d 4 f 5 l, 4 d 5 s 5 p, 4 d 5 s 5 d\right\}$ & $4 s^{1} 4 p^{5}+\left\{4 d^{2}, 4 d 4 f, 4 f^{2}, 4 d 5 l, 4 f 5 l, 5 s 5 l, 5 p 5 l^{\prime}, 5 d 5 f, 5 d 5 g, 5 f 5 g\right\}$ \\
$4 s^{2} 4 p^{4}+\left\{4 d^{4}, 4 d^{3} 4 f, 4 d^{3} 5 l, 4 d^{2} 4 f^{2}\right\}$ & $4 p^{6}+\left\{4 d^{2}, 4 d 4 f, 4 d 5 l, 4 f 5 l\right\}$ \\
$4 s 4 p^{5} 4 d^{4}$ & \\
$T r i p l y$-excited configuration & Triply-excited configurations $^{2}$, \\
$4 s^{2} 4 p^{3} 4 d^{5}$ & $4 s^{2} 4 p^{3} 4 d^{3}, 4 s^{2} 4 p^{3} 4 d^{2} 4 f, 4 s 4 p^{4} 4 d^{3}, 4 s 4 p^{4} 4 d^{2} 4 f$ \\
\hline \hline
\end{tabular}

TABLE I. Configuration sets adopted for $\mathrm{Sn}^{12+}$ and $\mathrm{Sn}^{14+}$ in the non-LTE/LTE configuration-averaged (CA) calculations (see main text). Configurations are grouped according to excitation degree. Here, angular momenta $l=0-4(s-g), l^{\prime}=1-4(p-g)$ and $\tilde{l}=2-4$ $(d-g)$, respectively.

The CSD resulting from this calculation is shown in black in Fig. 1. Importantly, the relevant EUV-emitting $\mathrm{Sn}^{11+}-\mathrm{Sn}^{14+}$ ions are produced under such conditions, with $\mathrm{Sn}^{12+}$ and $\mathrm{Sn}^{13+}$ clearly the dominant species. The average charge state of this plasma is $\langle Z\rangle_{\text {non-LTE }}=12.6$. Also illustrated in Fig. 1 is an LTE CA calculation (shown in red) performed at $\rho=2 \times 10^{-4} \mathrm{~g} \mathrm{~cm}^{-3}$ and $T_{e}=26$ $\mathrm{eV}$. These calculations yield $\langle Z\rangle_{\mathrm{LTE}}=12.6$, identical to the non-LTE calculation. Also, we note that the CSDs are very nearly identical. An ionisation temperature of $T_{Z}=26 \mathrm{eV}$ is thus established. We note that the ratio $T_{e} / T_{Z}=36 / 26 \approx 1.4$ is larger than that obtained by the scaling law $T_{e} / T_{Z} \approx\left(1+\left(5.36 \times 10^{13}\right) T_{e}^{7 / 2} n_{e}^{-1}\right)^{0.215} \approx 1.2$ obtained by Busquet et al. [65]. Much better agreement with this scaling law is obtained in the Nd:YAG-relevant case considered in [13] $\left(n_{e}=1.27 \times 10^{20} \mathrm{~cm}^{-3}, T_{Z}=32\right.$ $\left.\mathrm{eV}, T_{e}=34.5 \mathrm{eV}\right)$, where $T_{e} / T_{Z}=34.5 / 32 \approx 1.08$ and the scaling law yields $T_{e} / T_{Z} \approx 1.02$.

It is interesting to consider why optimum agreement in CSDs is obtained for $T_{e}>T_{Z}$. Exact reproduction of an LTE CSD is possible using a $T_{e}=26 \mathrm{eV}$ non-LTE calculation, however one must include an external radiation field in the calculation characterised by $T_{r}=T_{e}$. In this way, all atomic processes are in detailed balance. However, as detailed above, our non-LTE calculations do not include an external radiation field. In order to match our present calculations with a $T_{Z}=26 \mathrm{eV}$ LTE calculation, we must therefore compensate for the missing (de)excitation and ionisation channels provided by photoexcitation and photoionisation by increasing $T_{e}$ above $T_{Z}$. The difference $T_{e}-T_{Z}$ is effectively a gauge for how close the system is to LTE conditions; in the work of Torretti et al. [13], for example, a value $T_{e}-T_{Z}=2.5$ $\mathrm{eV}$ (where $T_{e}=34.5 \mathrm{eV}$ and $T_{Z}=32 \mathrm{eV}$ ) highlights the dominance of collisional rates over radiative rates at near-LTE conditions $\left(n_{e}=1.27 \times 10^{20} \mathrm{~cm}^{-3}\right)$. The same cannot be said for the $\mathrm{CO}_{2}$-relevant conditions, where a value $T_{e}-T_{Z}=10 \mathrm{eV}$ is indicative of truly non-LTE conditions, i.e., collisional rates do not significantly outweigh radiative rates.

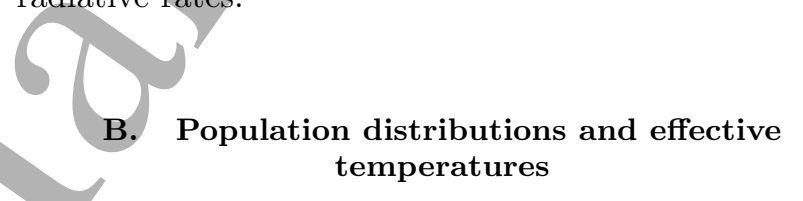

The replication of a non-LTE CSD using LTE computations, such as that shown in Fig. 1, is key for justifying the use of LTE opacities as an approximation for non-LTE opacities. An even stronger argument in favour of this approximation is to establish equivalence between the non-LTE and LTE-computed configuration populations (the cumulative sum of which yields the CSD - see Eq. (6)). To this end, we present in Fig. 2 non-LTE- and LTE-computed configuration populations $\left(P_{\text {non-LTE }}, P_{\mathrm{LTE}}\right)$ as a function of configuration-average energy $\left(E_{a v}\right)$ for $\mathrm{Sn}^{12+}$ (upper panel) and $\mathrm{Sn}^{14+}$ (lower panel). The non-LTE configuration population calculations are shown in Fig. 2 (a) $\left(\mathrm{Sn}^{12+}\right)$ and (d) $\left(\mathrm{Sn}^{14+}\right)$. The corresponding LTE calculations are shown in Fig. 2 (b) and (e). Configurations associated with single-, double- and triple-electron excitations are shown as blue, orange and green circles, respectively. The ground-state configurations, fixed at $E_{a v}=0 \mathrm{eV}$, are shown as gray circles. Perhaps the most striking feature of these calculations is the near-coincidence in non-LTE- and LTEcomputed configuration populations. This is quantified in Fig. 2 (c) and (f), where, for a given configuration $C$, we plot the non-LTE-normalised configuration population difference $(\mathrm{CPD})$ defined as $\left(P_{C, \text { non-LTE }}-\right.$ $\left.P_{C, \text { LTE }}\right) / P_{C, \text { non-LTE }}$. For the majority of configurations, this quantity takes on values below $1 \%$. This indicates a near-coincidence in non-LTE and LTE-computed configuration populations. The few configurations where the non-LTE-normalised CPD values are above $1 \%$ all have 

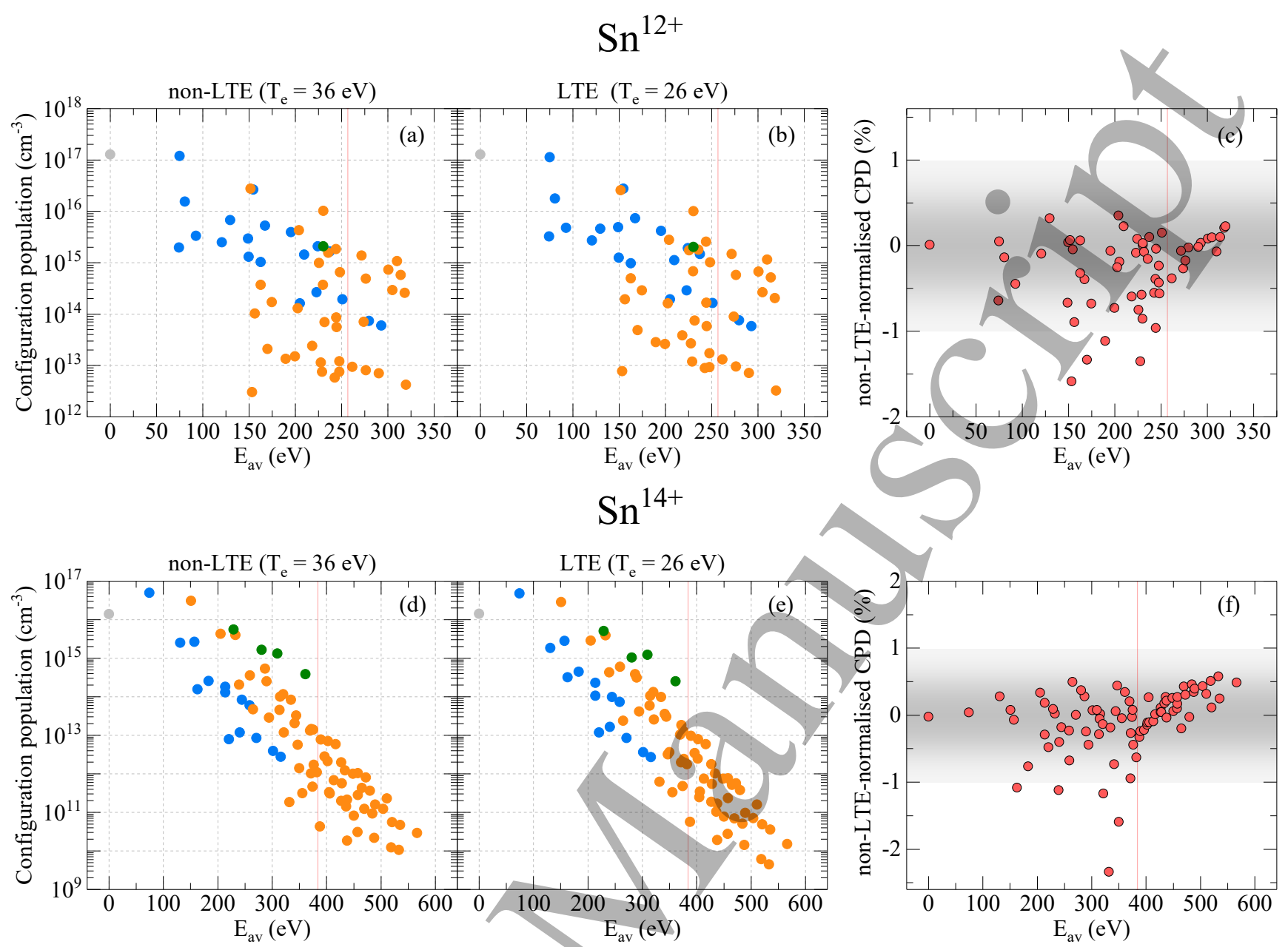

FIG. 2. Configuration populations for $\mathrm{Sn}^{12+}$ (top) and $\mathrm{Sn}^{14+}$ (bottom) calculated for a $T_{e}=36 \mathrm{eV}$ non-LTE plasma (panels (a) and (d)) and a $T_{e}=26 \mathrm{eV}$ LTE (panels (b) and (e)) plasma. The plasma mass density used in both calculations was $\rho=2 \times 10^{-4} \mathrm{~g} \mathrm{~cm}^{-3}$. Configurations associated with single-, double- and triple-electron excitations are shown as blue, orange and green circles, respectively. The ground-state configurations are shown in gray and are fixed at $E_{a v}=0 \mathrm{eV}$. Non-LTEnormalised configuration population differences (see text for description) are shown in panels (c) and (f). The vertical red lines indicate the ionisation potentials of $\mathrm{Sn}^{12+}$ and $\mathrm{Sn}^{144}$.

LTE-computed populations greater than the corresponding non-LTE populations.

In addition to validating the use of Busquet's approach in the current context, the equivalence in configuration populations reveals an interesting characteristic of the non-LTE-computed populations in that they appear to follow a single Boltzmann-esque temperature law of the form

$$
P_{C, \text { non-LTE }} \propto g_{C} \exp \left(-\frac{E}{T_{\text {eff }}}\right),
$$

where $g_{C}=\sum_{J_{C}}\left(2 J_{C}+1\right)$ is the total statistical weight of configuration $C\left(J_{C}\right.$ is an energy level associated with $C$ ) and $T_{\text {eff }}$ is a so-called effective temperature [67] (the Boltzmann constant $k_{B}$ typically appearing in equations of the form Eq. (8) is here "absorbed" into the definition of $\left.T_{\text {eff }}\right)$.

In order to evaluate $T_{\text {eff }}$, we plot in Fig. 3 the natural logarithm of the ratio $P_{C, \text { non-LTE }} / g_{C}$ as a function of $E_{a v}$ for (a) $\mathrm{Sn}^{12+}$ and (b) $\mathrm{Sn}^{14+}$. First-order polynomial fits to the data are shown in red. Excellent fits to the data are characterised by $R^{2}$ values of 0.99 attained in both cases. The effective temperatures $T_{\text {eff }}$ are obtained from the inverse-negative of the slopes of the lines of best fit. As indicated in the plots, the fits yield values of $T_{\text {eff }}=26.6\left(\mathrm{Sn}^{12+}\right)$ and $26.9 \mathrm{eV}\left(\mathrm{Sn}^{14+}\right)$, clearly very similar to the LTE ionisation temperature $T_{Z}=26 \mathrm{eV}$. This close agreement between $T_{\text {eff }}$ and $T_{Z}$ is perhaps not too surprising given the near-coincidence in non-LTE and LTE-computed configuration populations.

This observation, that non-LTE-computed configuration populations follow Boltzmann-like temperature laws has been studied extensively in the works of Bauche, 

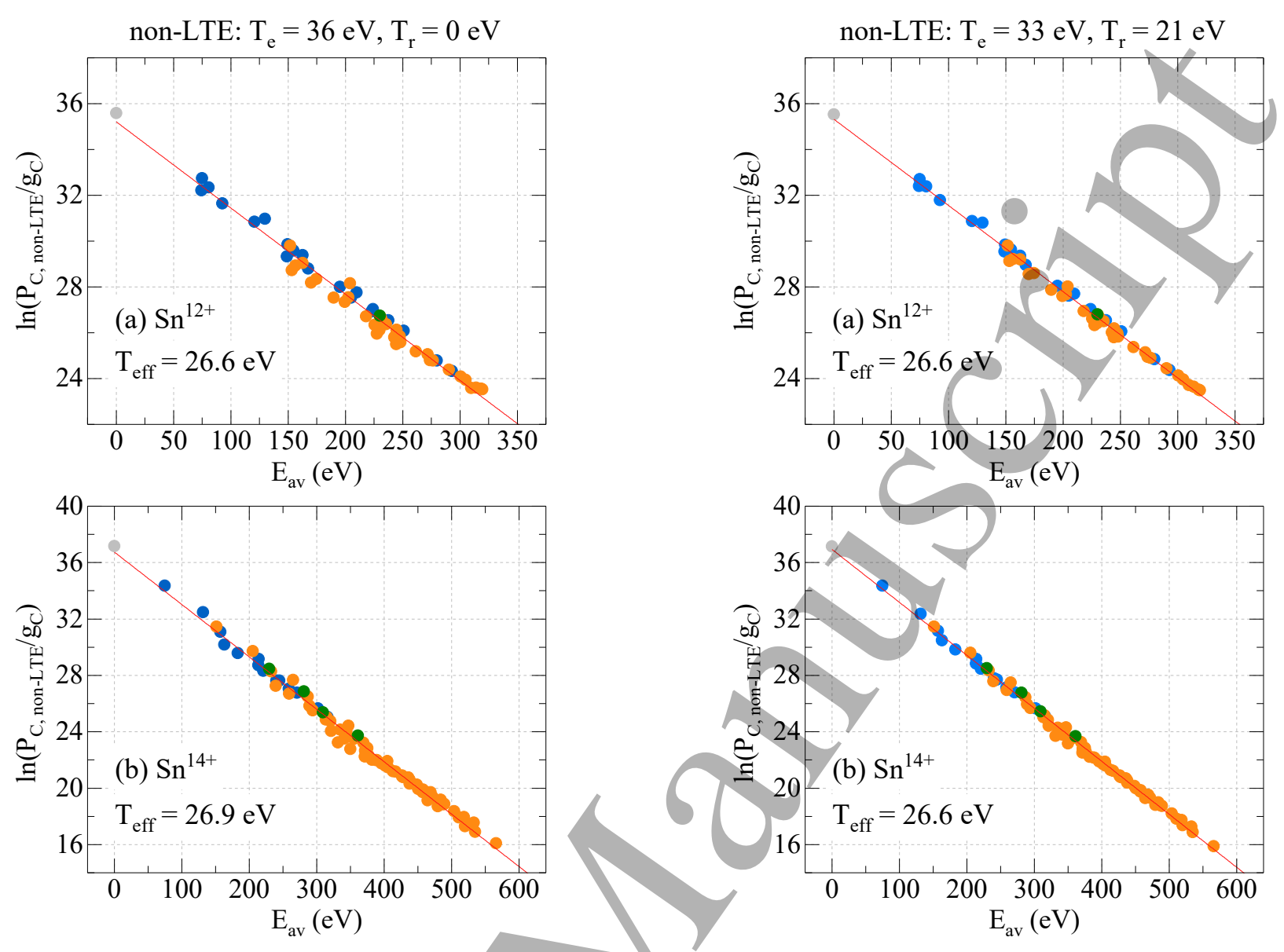

FIG. 3. Logarithm of the statistical-weight-normalised nonLTE-computed configuration populations for (a) $\mathrm{Sn}^{12+}$ and (b) $\mathrm{Sn}^{14+}$. The plasma conditions correspond to $\rho=2 \times 10^{-4}$ $\mathrm{g} \mathrm{cm}^{-3}, T_{e}=36 \mathrm{eV}$ and $T_{r}=0 \mathrm{eV}$. A first-order polynomial fit to the data is shown in red. Effective temperatures $T_{\text {eff }}$ are obtained from the slopes of the lines of best fit.

Bauche-Arnoult and others $[67-72]$. The emergence of temperature laws is attributed to the eomplex interplay of atomic processes defining the steady-state CR model. More specifically, the combination of (i) the microreversibility nature of collisional processes (which drives the population distributions towards LTE conditions) and (ii) correlation laws associated with spontaneous emission (high/low-lying upper/states typically decay towards high/low-lying lower states) tend to generate Boltzmann-type population distributions [71]. This is in fact a general phenomenon of non-LTE hot plasmas [68]. Finally, it should be noted that the approach of Bauche et al. [70], does not ascribe a single effective temperature $T_{\text {eff }}$ to a population distribution. Rather, configurations are grouped according to superconfigurations and Eq. (8) is used to derive superconfigurationspecific temperatures. These temperatures may vary significantly from superconfiguration-to-superconfiguration (see Fig. 1 of [70]). Although this approach differs to that
FIG. 4. Logarithm of the statistical-weight-normalised nonLTE-computed configuration populations for (a) $\mathrm{Sn}^{12+}$ and (b) $\mathrm{Sn}^{14+}$. The plasma conditions correspond to $\rho=2 \times 10^{-4}$ $\mathrm{g} \mathrm{cm}^{-3}, T_{e}=33 \mathrm{eV}$ and $T_{r}=21 \mathrm{eV}$. A first-order polynomial fit to the data is shown in red. Effective temperatures $T_{\text {eff }}$ are obtained from the slopes of the lines of best fit.

presented in Fig. 3 (where we have performed a "global" fit to all configurations), the non-LTE-computed population distributions are clearly well-described by a singletemperature Boltzmann-type law.

Finally, we wish to comment on the importance of a non-zero radiation field in our non-LTE computations. As outlined in Section III, all non-LTE calculations presented thus far have not included an external radiation field in the underlying atomic kinetics. Indeed, identifying an external radiation field appropriate for the current study is rather difficult as it is a purely non-local quantity, i.e., it depends on the radiative characteristics of the plasma surrounding the studied $\left(\rho, T_{e}\right)$ plasma. Proper evaluation of an external radiation field requires complex simulations involving the coupling of non-LTE atomic kinetics with a model for radiation transport in a multi- $\left(\rho, T_{e}, T_{r}\right)$ plasma. Such an endeavour is outside the scope of the present work. Instead, we have performed an additional non-LTE calculation where we 
have included a Planckian radiation field (characterised by a radiation field $T_{r}$ ). We fixed $T_{r}$ and tuned $T_{e}$ until Eq. (7) is established with a $T_{Z}=26 \mathrm{eV}$ LTE plasma. We find that a $T_{e}=33 \mathrm{eV}$ and $T_{r}=21 \mathrm{eV}$ plasma, for example, reproduces both Eq. (7) and the LTE CSD. Moreover, the configuration populations associated with $\mathrm{Sn}^{12+}$ and $\mathrm{Sn}^{14+}$ are also found to be in excellent agreement with the LTE-computed configuration populations. This is demonstrated in Fig. 4, where, in a similar vein to Fig. 3, we plot the natural logarithm of the ratio $P_{C \text {,non-LTE }} / g_{C}$ as a function of $E_{a v}$ for (a) $\mathrm{Sn}^{12+}$ and (b) $\mathrm{Sn}^{14+}$. Effective temperatures obtained from the slopes of the lines of best fit (shown in red) are $T_{\text {eff }}=26.6 \mathrm{eV}$ for both $\mathrm{Sn}^{12+}$ and $\mathrm{Sn}^{14+}$, clearly in excellent agreement with the data presented in Fig. 3. The introduction of a non-zero radiation field in these computations therefore does not break the observed Boltzmann-esque temperature law once equivalence between the non-LTE and LTE-computed CSDs is established.

\section{TIN-ION OPACITIES}

We now present the results of detailed, level-resolved LTE opacity calculations at $\mathrm{CO}_{2}$ laser-driven plasma conditions. The LTE plasma conditions considered here are the same as those of Section III $\left(\rho=2 \times 10^{-4} \mathrm{~g}\right.$ $\left.\mathrm{cm}^{-3}, T_{Z}=26 \mathrm{eV}\right)$. Unlike Section III, we adopt a fully level-resolved approach where ATOMIC is used to calculate level populations and bound-bound opacities. The atomic structures employed in these calculations are the same as those considered in Ref. [13] (see section II).

The results of these calculations are presented in Fig. 5. Panels (a) - (d) illustrate the contributions to the opacity from the four charge states $\mathrm{Sn}^{11+}-\mathrm{Sn}^{14+}$. In a similar fashion to Torretti et al. [13], the different contributions to the opacity are plotted in a cumulative fashion; photoabsorption from levels in the ground manifold to singly-excited levels are shown in blue (we call this the "ground opacity"); the summation of photoabsorption from singly-excited levels to doubly-excited levels ("first opacity") and the ground opacity is shown by the line bounding the orange shaded area; the summation of photoabsorption from doubly- and triply-excited levels ("higher opacity"), the ground opacity and first opacity is shown by the line bounding the green shaded area. The grey region in each panel represents a $2 \%$ bandwidth centered at $13.5 \mathrm{~nm}$.

First, examining the ordinate of Fig. 5 (b) and (c), one readily concludes that $\mathrm{Sn}^{12+}$ and $\mathrm{Sn}^{13+}$ are the dominant contributors to the opacity in this plasma. This can be understood from the CSD presented in Fig. 1, where these two charge states accumulate nearly $80 \%$ of the total ion population. Although the majority of the opacity in the $\mathrm{Sn}^{12+}$ ion can be attributed to the ground opacity, the first and higher opacities contribute to the spectrum at wavelengths larger than $13.5 \mathrm{~nm}$. The same is true for $\mathrm{Sn}^{114}$. One of the most striking features of these opac-
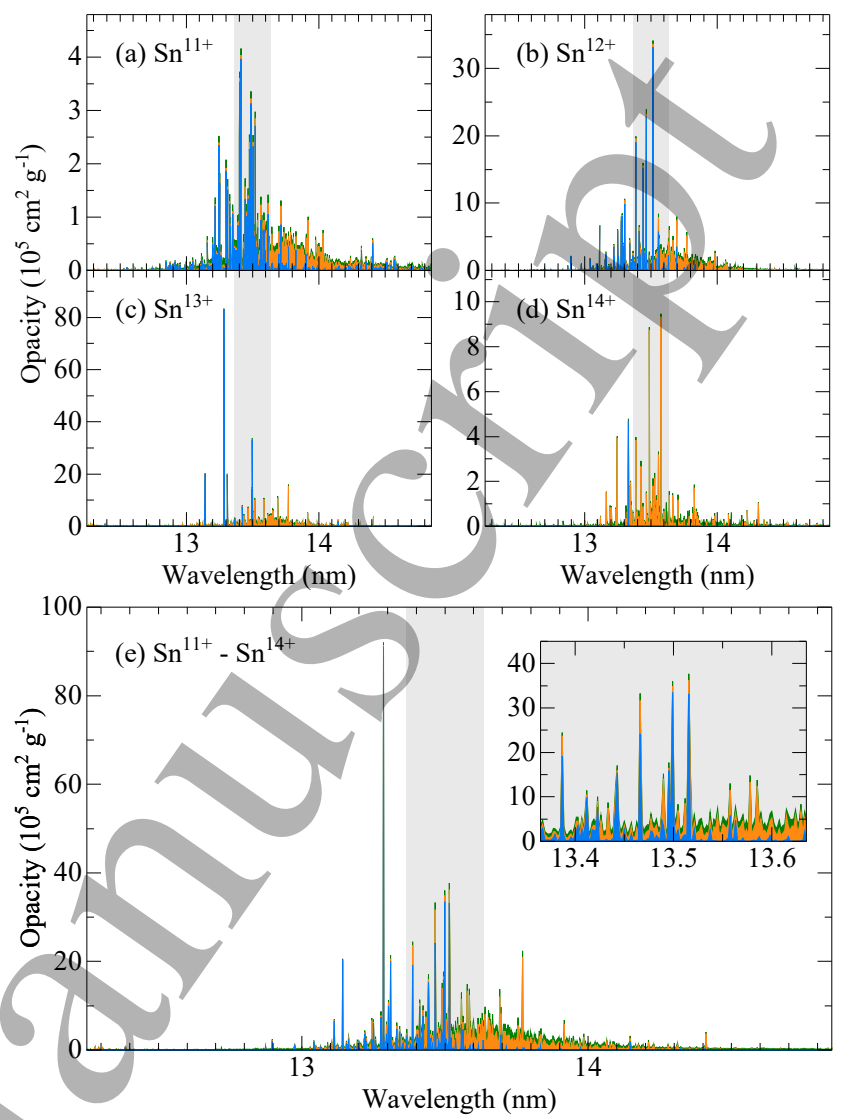

FIG. 5. Panels (a) - (d): Opacities of the ions $\mathrm{Sn}^{11+}-\mathrm{Sn}^{14+}$. The opacities are plotted in a cumulative fashion. Photoabsorption from levels of the ground manifold to singly-excited levels is shown in blue ("ground opacity"). The summation of photoabsorption from singly-excited levels to doubly-excited levels ("first opacity") and the ground opacity is shown by the line bounding the orange shaded region. The line bounding the green shaded region is the summation of photoabsorption from doubly- and triply-excited levels ("higher opacity"), the ground opacity and first opacity. The total opacity, obtained by summing the contributions of $\mathrm{Sn}^{11+}-\mathrm{Sn}^{14+}$, is shown in panel (e). The inset in panel (e) shows the opacity in a $2 \%$ bandwidth centered at $13.5 \mathrm{~nm}$.

ity spectra is the spectral dominance of a transition in $\mathrm{Sn}^{13+}$ (see Fig. 5 (c)). This transition is the subvalence excitation $4 p^{6} 4 d^{2} D_{5 / 2}-4 p^{5} 4 d^{2}{ }^{2} F_{7 / 2}$. It has a calculated wavelength of $13.286 \mathrm{~nm}$, in good agreement with experimental identifications of this transition at 13.3014 [17], 13.3020 [73] and $13.301 \mathrm{~nm}$ [29]. The opacity of this transition is $\kappa \approx 83 \times 10^{5} \mathrm{~cm}^{2} \mathrm{~g}^{-1}$, nearly a factor 3 higher than any other transition in $\mathrm{Sn}^{11+}-\mathrm{Sn}^{14+}$. There are two reasons for this spectral dominance. First, as illustrated in Fig. 2, a significant fraction of $\mathrm{Sn}^{12+}$ and $\mathrm{Sn}^{14+}$ ions are in their ground-state configurations. This also true for $\mathrm{Sn}^{13+}$. Secondly, this $L S$-allowed transition exhibits a large oscillator strength. This combination, which appears multiplicatively in the first term of Eq. (2), is the origin of this large opacity value. As in the 


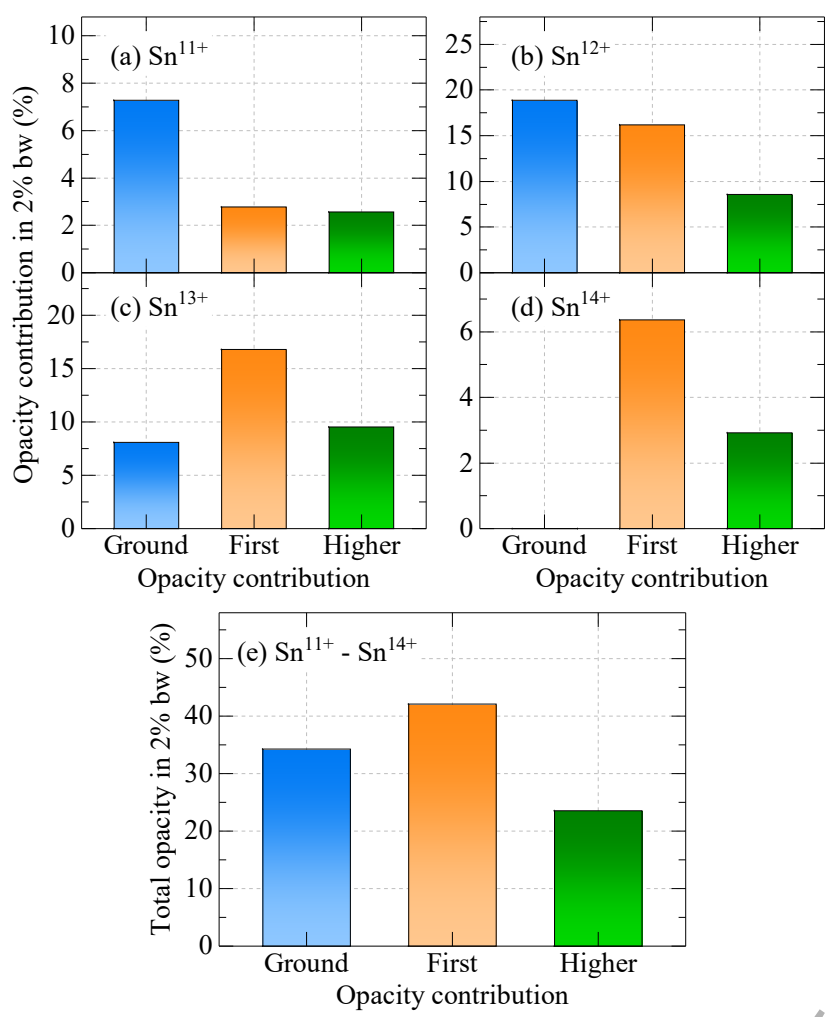

FIG. 6. Panels (a) - (d): Contributions from the ground, first and higher opacities in the $2 \%$ bandwidth (bw) in $\mathrm{Sn}^{11+}$ $\mathrm{Sn}^{14+}$. The total opacity in the $2 \%$ bandwidth, obtained by summing the contributions in panels $(\mathrm{a})-(\mathrm{d})$, is shown in panel (e).

case of $\mathrm{Sn}^{11+}$ and $\mathrm{Sn}^{12+}$, higher-order opacities are seen to contribute at wavelengths above $13.5 \mathrm{~nm}$, however only on the order $\kappa \approx 10 \times 10^{5} \mathrm{~cm}^{2} \mathrm{~g}^{-1}$. Moving to the closed $4 p$ subshell $\mathrm{Sn}^{14+}$ ion we observe an interesting shift in opacity dominance away from the ground opacity to the first opacity. The ground opacity in this wavelength region is dominated by the transition $4 p^{6}{ }^{1} S_{0}-4 p^{5} 4 d^{1} P_{1}$, which has a calculated wavelength of $13.332 \mathrm{~nm}$. This is in excellent agreement with experimental identifications of this transition at $\lambda=13.344 \mathrm{~nm}$ [29] and $\lambda=13.343$ $[18,74]$. This transition is clearly much weaker than the $4 p^{6} 4 d^{2} D_{5 / 2}-4 p^{5} 4 d^{2}{ }^{2} F_{7 / 2}$ transition in $\mathrm{Sn}^{13+}$. This is because (i) the $4 p^{6}{ }^{1} S_{0}$ level in $\mathrm{Sn}^{14+}$ has a smaller population than the $4 p^{6} 4 d^{2} D_{5 / 2}$ level in $\mathrm{Sn}^{13+}$ and (ii) the transition in $\mathrm{Sn}^{14+}$ involves energy levels having low angular momenta $\left(J_{\text {lower,upper }}=0,1\right)$ and therefore has a lower oscillator strength than the aforementioned transition in $\mathrm{Sn}^{13+}$. As is clear from Fig. 5 (d), photoabsorption from singly-excited levels is the main source of opacity in $\mathrm{Sn}^{14+}$. Importantly, all of the opacity in the industrially-relevant $2 \%$ bandwidth (the gray shaded region) in $\mathrm{Sn}^{14+}$ originates from higher-order opacities, the first opacity being the dominant contributor.

The total opacity, obtained by summing the contri-

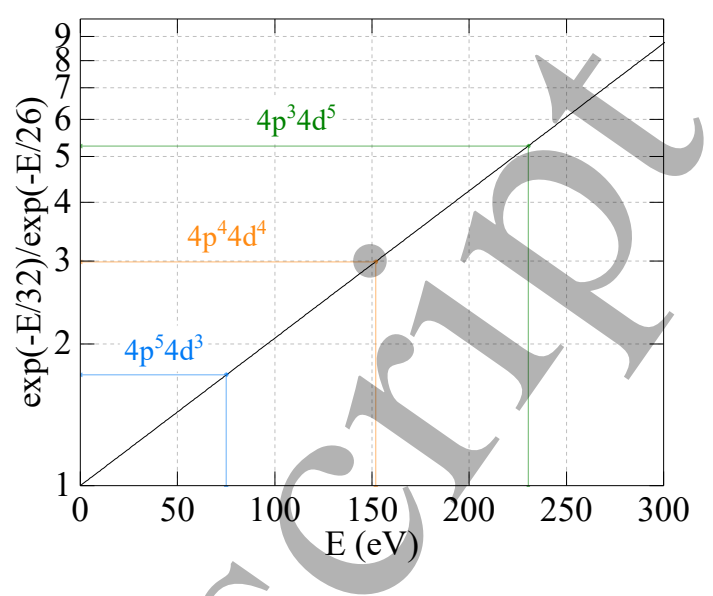

FIG. 7. Ratio of the partition function factor for $T_{e}=32$ and $26 \mathrm{eV}$ plasmas (shown in black). The ratio for the $4 p^{5} 4 d^{3}$, $4 p^{4} 4 d^{4}$ and $4 p^{3} 4 d^{5}$ configurations in $\mathrm{Sn}^{12+}$ are shown in blue, orange and green, respectively.

butions in Fig. 5 (a) - (d), is shown in Fig. 5 (e). As expected, most of the higher-order opacity contributions are located at wavelengths above $13.5 \mathrm{~nm}$. The inset illustrates the contributions to the opacity in the $2 \%$ bandwidth. Higher-order opacities clearly make a significant contribution to this industrially-relevant spectral region. In essence, they tend to "fill out" regions between the ground opacities. The charge-state-specific opacity contributions in the $2 \%$ bandwidth are illustrated in Fig. 6 (a) - (d), with their combined opacity contribution illustrated in Fig. 6 (e). Importantly, only $34 \%$ of the opacity arises from the ground opacity. Of the remaining $66 \%$, approximately $42 \%$ originates from the first opacity and $24 \%$ is associated with the higher opacity. This result clearly highlights the important spectral role played by multiply-excited transitions in $\mathrm{CO}_{2}$ laser-driven plasma conditions.

Finally, we wish to compare these results with the Nd:YAG-relevant case considered in [13]. The plasma conditions considered in Ref. [13] were $\rho=2 \times 10^{-3}$ $\mathrm{g} \mathrm{cm}^{-3}$ and $T_{e}=32 \mathrm{eV}$. This LTE calculation yielded $n_{e}=1.27 \times 10^{20} \mathrm{~cm}^{-3}$ (an order of magnitude higher than the current case) and an average charge state $\langle Z\rangle$ similar to the present case. Comparing Fig. 5 (e) with Fig. 3 (e) of [13], we see that, relative to the ground opacity, the higher-order opacities in the Nd:YAG case make a significantly larger contribution than in the present case. Comparing Maxwell-Boltzmann distributions at $T_{e}=32 / 26$ $\mathrm{eV}$, one observes that the former has a larger fraction of higher-energy electrons which can collisionally populate high-lying levels associated with doubly-, triply- and quadruply-excited configurations. As both calculations were performed in LTE, the populations of the levels are described by the so-called "partition function factor", which for some level $J$ is written $g_{J} \exp \left(-E_{J} / T_{e}\right)$. To enable a comparison between both cases, we plot in Fig. 7 
the ratio of the partition function factor for $T_{e}=32$ and $26 \mathrm{eV}$ (shown in black) using a continuous energy variable $E$. The statistical weight $g_{C}$ naturally drops out of this ratio. It is clear from this figure that the population of multiply-excited (doubly-, triply- and quadruply-excited) states is much higher in the $T_{e}=32 \mathrm{eV}$ plasma than in the $T_{e}=26 \mathrm{eV}$ plasma. Examples of this ratio for the $4 p^{5} 4 d^{3}, 4 p^{4} 4 d^{4}$ and $4 p^{3} 4 d^{5}$ configurations in $\mathrm{Sn}^{12+}$ are shown in blue, orange and green, respectively. Interestingly, the increased population of the multiply-excited states in the Nd:YAG-relevant plasma is a natural byproduct of the higher temperatures needed to generate a CSD equivalent to that generated under relevant $\mathrm{CO}_{2-}$ laser-driven tin plasma conditions.

\section{CONCLUSION}

In conclusion, we have investigated the role played by multiply-excited states in industrially-relevant $\mathrm{CO}_{2}$ laser-driven tin plasma conditions. First, we employed Busquet's ionisation temperature method to match the charge state distribution of a non-LTE plasma with an LTE computation. We find excellent agreement between the non-LTE-computed configuration populations and LTE calculations for both $\mathrm{Sn}^{12+}$ and $\mathrm{Sn}^{14+}$. Moreover, we identify the existence of Boltzmann-like temperature laws in the non-LTE population distributions. In the second part of this study we performed detailed, levelresolved LTE opacity calculations at plasma conditions pertaining to $\mathrm{CO}_{2}$ laser-driven plasma conditions. Approximately $66 \%$ of the opacity in the $2 \%$ bandwidth centered at $13.5 \mathrm{~nm}$ was found to arise from transitions between multiply-excited states. This result highlights the important role played by multiply-excited states in industrially-relevant plasma conditions. This work paves the way for the generation of detailed LTE opacity tables at numerous $\left(\rho, T_{e}, T_{r}\right)$ non-LTE plasma conditions for incorporation in radiation-hydrodynamic simulations. Such simulations will enable reliable predictions of EUV emission from LPPs and are therefore key for guiding experimental efforts in characterising and optimising laserdriven plasma sources of EUV light.

\section{ACKNOWLEDGMENTS}

We would like to thank W. van der Zande, W. Ubachs and R. Hoekstra for their useful comments on the paper. This project has received funding from European Research Council (ERC) Starting Grant number 802648 and is part of the VIDI research programme with project number 15697, which is financed by the Netherlands Organization for Scientific Research (NWO). Part of this work has been carried out at the Advanced Research Center for Nanolithography (ARCNL), a public-private partnership of the University of Amsterdam (UvA), the Vrije Universiteit Amsterdam (VU), NWO and the semiconductor equipment manufacturer ASML. Part of this work was supported by the ASC PEM program of the US Department of Energy through the Los Alamos National Laboratory. Los Alamos National Laboratory is operated by Triad National Security, LLC, for the National Nuclear Security Administration of U.S. Department of Energy (Contract No. 89233218NCA000001).

\section{REFERENCES}

[1] D. Bleiner, J. Costello, F. Dortan, G. O'Sullivan, L. Pina, and A. Michette, eds., Short Wavelength Laboratory Sources: Principles and Practices (The Royal Society of Chemistry, London, 2014).

[2] G. Schneider, P. Guttmann, S. Heim, S. Rehbein, F. Mueller, K. Nagashima, J. Bernard Heymann, W. G. Müller, and J. G. McNally, Nat. Methods 7, 985 (2010).

[3] G. McDermott, M. A. Le Gros, C. G. Knoechel, M. Uchida, and C. A. Larabell, Trends Cell Biol. 19, 587 (2009).

[4] T. Helk, M. Zürch, and C. Spielmann, Struct. Dyn. 6, 010902 (2019).

[5] A. Sakdinawat and D. Attwood, Nat. Photon. 4, 840 (2010).

[6] A. Bartnik, H. Fiedorowicz, R. Jarocki, J. Kostecki, M. Szczurek, and P. Wachulak, Nuclear Instruments and Methods in Physics Research Section A: Accelerators, Spectrometers, Detectors and Associated Equipment 647, 125 (2011).

[7] C. Mack, Fundamental Principles of Optical Lithography: The Science of Microfabrication (John Wiley \& Sons Ltd,
West Sussex, 2007).

[8] C. M. Garner, Philosophical Transactions of the Royal Society A: Mathematical, Physical and Engineering Sciences 370, 4015 (2012).

[9] E. van Setten, G. Schiffelers, E. Psara, D. Oorschot, N. Davydova, J. Finders, L. Depre, and V. Farys, in 30th European Mask and Lithography Conference, Vol. 9231, edited by U. F. W. Behringer, International Society for Optics and Photonics (SPIE, 2014) pp. $50-63$.

[10] C. Wagner and N. Harned, Nat. Photon. 4, 24 (2010).

[11] V. Bakshi, ed., EUV Lithography (SPIE Press, Washington, 2009).

[12] V. Bakshi, ed., EUV Lithography, Second Edition (SPIE Press, Washington, 2018).

[13] F. Torretti, J. Sheil, R. Schupp, M. M. Basko, M. Bayraktar, R. A. Meijer, S. Witte, W. Ubachs, R. Hoekstra, O. O. Versolato, A. J. Neukirch, and J. Colgan, Nat. Commun. 11, 2334 (2020).

[14] I. Fomenkov, D. Brandt, A. Ershov, A. Schafgans, Y. Tao, G. Vaschenko, S. Rokitski, M. Kats, M. Vargas, M. Purvis, et al., Adv. Opt. Techn. 6, 173 (2017). 
[15] M. Purvis, I. V. Fomenkov, A. A. Schafgans, M. Vargas, S. Rich, Y. Tao, S. I. Rokitski, M. Mulder, E. Buurman, M. Kats, J. Stewart, A. D. LaForge, C. Rajyaguru, G. Vaschenko, A. I. Ershov, R. J. Rafac, M. Abraham, D. C. Brandt, and D. J. Brown, in Extreme Ultraviolet (EUV) Lithography IX, Vol. 10583, edited by K. A. Goldberg, International Society for Optics and Photonics (SPIE, 2018) pp. $476-485$.

[16] S. S. Churilov and A. N. Ryabtsev, Phys. Scr. 73, 614 (2006).

[17] S. S. Churilov and A. N. Ryabtsev, Opt. Spectrosc. 101, 169 (2006).

[18] A. N. Ryabtsev, É. Y. Kononov, and S. S. Churilov, Opt. Spectrosc. 105, 844 (2008).

[19] G. O'Sullivan, B. Li, R. D'Arcy, P. Dunne, P. Hayden, D. Kilbane, T. McCormack, H. Ohashi, F. O'Reilly, P. Sheridan, E. Sokell, C. Suzuki, and T. Higashiguchi, J. Phys. B 48, 144025 (2015).

[20] O. O. Versolato, Plasma Sources Sci. Technol. 28, 083001 (2019).

[21] M. Richardson, C.-S. Koay, K. Takenoshita, C. Keyser, and M. Al-Rabban, Journal of Vacuum Science \& Technology B: Microelectronics and Nanometer Structures Processing, Measurement, and Phenomena 22, 785 (2004).

[22] N. M. Felix and D. T. Attwood, in Extreme Ultraviolet (EUV) Lithography XI, Vol. 11323, edited by N. M. Felix and A. Lio, International Society for Optics and Photonics (SPIE, 2020).

[23] G. O'Sullivan and P. K. Carroll, J. Opt. Soc. Am. 71, 227 (1981).

[24] P. Mandelbaum, M. Finkenthal, J. L. Schwob, and M. Klapisch, Phys. Rev. A 35, 5051 (1987).

[25] W. Svendsen and G. O'Sullivan, Phys. Rev. A 50, 3710 (1994).

[26] H. Ohashi, S. Suda, H. Tanuma, S. Fujioka, H. Nishimura, A. Sasaki, and K. Nishihara, J. Phys. B: At. Mol. Opt. Phys. 43, 065204 (2010).

[27] R. D'Arcy, H. Ohashi, S. Suda, H. Tanuma, S. Fujioka, H. Nishimura, K. Nishihara, C. Suzuki, T. Kato, F. Koike, J. White, and G. O'Sullivan, Phys. Rey. A 79, 042509 (2009).

[28] R. D'Arcy, O. Morris, H. Ohashi, S. Suda, H. Tanuma, S. Fujioka, H. Nishimura, K. Nishihara, C. Suzuki, T. Kato, F. Koike, and G. O'Sullivan, Physica Scripta T144, 014026 (2011).

[29] J. Scheers, C. Shah, A. Ryabtsev, H. Bekker, F. Torretti, J. Sheil, D. A. Czapski, J. C. Berengut, W. Ubachs, J. R. C. López-Urrutia, R. Hoekstra, and O. O. Versolato, Phys. Rev. A 101, 062511 (2020).

[30] V. Bakshi, ed., EUV Sources for Lithography (SPIE Press, Washington, 2006).

[31] J. White, P. Hayden, P. Dunne, A. Cummings, N. Murphy, P. Sheridan, and G. O'Sullivan, Journal of Applied Physics 98, 113301 (2005).

[32] S. Fujioka, H. Nishimura, K. Nishihara, A. Sasaki, A. Sunahara, T. Okuno, N. Ueda, T. Ando, Y. Tao, Y. Shimada, K. Hashimoto, M. Yamaura, K. Shigemori, M. Nakai, K. Nagai, T. Norimatsu, T. Nishikawa, N. Miyanaga, Y. Izawa, and K. Mima, Phys. Rev. Lett. 95, 235004 (2005).

[33] M. Poirier, T. Blenski, F. de Gaufridy de Dortan, and F. Gilleron, J. Quant. Spectrosc. Radiat. Transfer 99,
482 (2006).

[34] J. J. MacFarlane, C. L. Rettig, P. Wang, I. E. Golovkin, and P. R. Woodruff, in Emerging Lithographic Technologies IX, Vol. 5751, edited by R. S. Mackay, International Society for Optics and Photonics (SPIE, 2005) pp. $588-$ 600.

[35] F. Koike and S. Fritzsche, Radiation Physics and Chemistry 76, 404 (2007).

[36] F. de Gaufridy de Dortan, J. Phys. B 40, 599 (2007).

[37] A. Sasaki, A. Sunahara, H. Furukawa, K. Nishihara, S. Fujioka, T. Nishikawa, F. Koike, H. Ohashi, and H. Tanuma, J. Appl. Phys. 107, 113303 (2010).

[38] J. Zeng, C. Gao, and J. Yuan, Phys. Rev. E 82, 026409 (2010).

[39] J. Colgan, D. P. Kilcrease, J. Abdallah, M. E. Sherrill, C. J. Fontes, P. Hakel, and G. S. J. Armstrong, High Energy Density Phys. 23, 133 (2017).

[40] A. Sasaki, A. Sunahara, K. Nishihawra, T. Nishikawa, F. Koike, and H. Tanuma, Journal of Physics: Conference Series 112, 042062 (2008).

[41] A. Sasaki, A. Sunahara, K. Nishihara, T. Nishikawa, K. Fujima, T. Kagawa, F. Koike, and H. Tanuma, High Energy Density Physics 3, 250 (2007).

[42] C. J. Fontes, H. L. Zhang, J. Abdallah Jr, R. E. H. Clark, D. P. Kilcrease, J. Colgan, R. T. Cunningham, P. Hakel, N. H. Magee, and M. E. Sherrill, J. Phys. B: At. Mol. Opt. Phys. 48, 144014 (2015).

[43] N. H. Magee, J. Abdallah, J. Colgan, P. Hakel, D. P. Kilcrease, S. Mazevet, M. Sherrill, C. J. Fontes, and H. Zhang, in AIP Conf. Proc., Vol. 730 (AIP, 2004) pp. 168-179.

[44] P. Hakel, M. E. Sherrill, S. Mazevet, J. Abdallah Jr, J. Colgan, D. P. Kilcrease, N. H. Magee, C. J. Fontes, and H. L. Zhang, J. Quant. Spectrosc. Radiat. Transf. 99, 265 (2006).

[45] J. J. Abdallah, R. E. H. Clark, and R. D. Cowan, "Theoretical atomic physics code development Vol. I CATS: Cowan Atomic Structure Code, Los Alamos National Laboratory Report LA-11436-M, December 1988 (unpublished)," (1988).

[46] R. D. Cowan, The Theory of Atomic Structure and Spectra (University of California Press, Berkeley, 1981).

[47] A. Kramida, Atoms 7, 64 (2019).

[48] J.-F. Wyart, Canadian Journal of Physics 89, 451 (2011).

[49] A. Ryabtsev, E. Kononov, R. Kildiyarova, W.-U. L. Tchang-Brillet, J.-F. Wyart, N. Champion, and C. Blaess, Atoms 3, 273 (2015).

[50] A. Windberger, F. Torretti, A. Borschevsky, A. Ryabtsev, S. Dobrodey, H. Bekker, E. Eliav, U. Kaldor, W. Ubachs, R. Hoekstra, J. R. Crespo López-Urrutia, and O. O. Versolato, Phys. Rev. A 94, 012506 (2016).

[51] F. Torretti, A. Windberger, A. Ryabtsev, S. Dobrodey, H. Bekker, W. Ubachs, R. Hoekstra, E. V. Kahl, J. C. Berengut, J. R. Crespo López-Urrutia, and O. O. Versolato, Phys. Rev. A 95, 042503 (2017).

[52] I. Hubeny and D. Mihalas, Theory of Stellar Atmospheres: An Introduction to Astrophysical Nonequilibrium Quantitative Spectroscopic Analysis (Princeton University Press, Princeton, 2015).

[53] J. Castor, Radiation Hydrodynamics (Cambridge University Press, Cambridge, 2004).

[54] Y. Ralchenko, Plasma and Fusion Research 8, 2503024 (2013). 
[55] H. A. Scott, in Modern Methods in Collisional-Radiative Modeling of Plasmas, edited by Y. Ralchenko (Springer International Publishing, Cham, 2016) pp. 81-104.

[56] P. Hakel and D. P. Kilcrease, in AIP Conf. Proc., Vol. 730 (AIP, 2004) pp. 190-199.

[57] D. P. Kilcrease, J. Colgan, P. Hakel, C. J. Fontes, and M. E. Sherrill, High Energy Density Physics 16, 36 (2015).

[58] C. J. Fontes, J. Abdallah Jr, R. E. H. Clark, and D. P. Kilcrease, J. Quant. Spectrosc. Radiat. Transf. 65, 223 (2000).

[59] C. J. Fontes, J. Colgan, and J. Abdallah Jr., in Modern Methods in Collisional-Radiative Modeling of Plasmas, edited by Y. Ralchenko (Springer International Publishing, Cham, 2016) pp. 17-50.

[60] S. J. Rose, J. Quant. Spectrosc. Radiat. Transf. 54, 333 (1995).

[61] R. Rodríguez, G. Espinosa, and J. M. Gil, Phys. Rev. E 98, 033213 (2018).

[62] R. E. H. Clark, J. Abdallah Jr., and J. B. Mann, Astrophys. J. 381, 597 (1991).

[63] M. Lysaght, D. Kilbane, N. Murphy, A. Cummings, P. Dunne, and G. O'Sullivan, Phys. Rev. A 72, 014502
(2005).

[64] M. Busquet, Phys. of Fluids B 5, 4191 (1993).

[65] M. Busquet, D. Colombant, M. Klapisch, D. Fyfe, and J. Gardner, High Energy Density Physics 5, 270 (2009).

[66] M. Klapisch and A. Bar-Shalom, J. Quant. Spectrosc. Radiat. Transf. 58, 687 (1997).

[67] J. Bauche and C. Bauche-Arnoult, J. Phys. B 33, L283 (2000).

[68] K. B. Fournier, J. Bauche, and C. Bauche-Arnoult, J. Phys. B 33, 4891 (2000).

[69] C. Bauche-Arnoult and J. Bauche, J. Quant. Spectrosc. Radiat. Transf. 71, 189 (2001).

[70] J. Bauche, C. Bauche-Arnoult, and K. B. Fournier, Phys. Rev. E 69, 026403 (2004).

[71] J. Bauche, C. Bauche-Arnoult, and O. Peyrusse, J. Quant. Spectrosc. Radiat. Transf. 99, 55 (2006).

[72] M. Busquet, J. Quant. Spectrosc. Radiat. Transf. 99, 131 (2006).

[73] J. Sugar, V. Kaufman, and W. L. Rowan, J. Opt. Soc. Am. B 9, 1959 (1992).

[74] J. Sugar, V. Kaufman, and W. L. Rowan, J. Opt. Soc. Am. B 8, 2026 (1991). 